\title{
UP TO DATE SULLA SIFILIDE: PATOLOGIA RIEMERGENTE, RITORNA L'ATTUALITÀ DI UNO SCREENING
}

\section{Gasponi A., Panuccio A.}

\author{
Lab. Analisi Osp. "Bellaria Bologna \\ U.O. Immunologia e Vir. Lab. Sanita' Pubblica Milano
}

La sifilide ha conosciuto nel corso della storia cicliche ricorrenze ma, con l'avvento dell'era antibiotica, a partire dalla metà del secolo scorso, si è assistito in tutto il mondo occidentale ad un calo verticale dei casi di sifilide e la malattia sembrava destinata, se non proprio a scomparire, a mantenersi tuttavia su livelli di incidenza assai ridotti.

Hanno destato pertanto sorpresa le segnalazioni provenienti da diversi paesi dell'Europa occidentale concernenti un aumento dei casi di sifilide.

Nel periodo 2001-2004 sono stati descritti trends in aumento in Belgio, Francia, Germania, Irlanda, Cecoslovacchia, Gran Bretagna, Danimarca, Italia.

In tutti questi paesi il gruppo maggiormente colpito è risultato essere quello dei maschi omosessuali (MSM, Men with Sex Men) e il fattore di rischio preponderante è rappresentato dalla promiscuità sessuale ( prostitute e scambisti nei sempre più diffusi sexual networks). La terapia HAART in uso dal 1996 negli individui infetti con HIV, oltre a produrre una più elevata proporzione di popolazione omosessuale attiva ha notevolmente innalzato i casi di coinfezione HIV-LUE. Nondimeno ha influito, il crescente afflusso di immigrati provenienti da aree geografiche in cui si registrano già da tempo alti tassi di incidenza e prevalenza (paesi dell' Est Europeo).

I fattori che influenzano la trasmissione della sifilide sono molti e, conseguentemente, gli interventi di prevenzione e controllo della malattia devono attuarsi a più livelli.

Ai metodi di ricerca tradizionali (VDRL, TPHA ed FTA) si sono affiancati negli ultimi anni, nuove generazioni di tests sierologici come ELISA ed Immunoblotting che utlizzando "antigeni da ricombinante" rendono la diagnosi sierologica più agevole e soddisfacente sia in termini di sensibilità che di specificità.

Tuttavia, dall'esame dei reports provenienti dai paesi sopraccitati emerge l'importanza di migliorare la sorveglianza come momento chiave nel rafforzamento degli interventi di prevenzione e di controllo. Viene discussa la reale utilità dei programmi di screening (generalizzati o focalizzati su gruppi di persone a rischio) in rapporto a una valutazione costo/beneficio. 